\title{
Synthesis and Characterization of NIR Dye-Doped Nanoparticles for in Vivo Tumor Diagnostics
}

\author{
Christine Schneider ${ }^{1}$, Sofia Dembski ${ }^{2,3}$ \\ ${ }^{1}$ University Hospital of Wuerzburg \\ Josef-Schneider-Str. 2, 97080 Wuerzburg, Germany \\ christine.b.schneider@uni-wuerzburg.de \\ ${ }^{2}$ Fraunhofer Institute for Silicate Research Institute ISC \\ Neunerplatz 2, 97082 Wuerzburg, Germany \\ ${ }^{3}$ Translational Center Wuerzburg, Regenerative Therapies in Oncology and Musculoskeletal Diseases \\ Roentgenring 11, 97070 Wuerzburg, Germany \\ sofia.dembski@fraunhofer.isc.de
}

\section{Extended Abstract}

Nanoparticles (NPs) are promising tools for a wide spectrum of biological and medical applications. They can be used as carrier and delivery systems for active agents such as biomolecules, dyes and a wide range of sensitive substances and also contribute to the stabilization of these compounds in vivo. Real time non-destructive imaging screening in vivo can be performed by means of fluorescent based methods. Near infrared (NIR) dyes are perfectly suited for this purpose. They are very promising for tissue labeling because of the fact that in the IR range there is significantly lower background fluorescence than in the visible range. Another feature of tissue is the so called transparent "NIR-window" at wavelengths from $650 \mathrm{~nm}$ to $1350 \mathrm{~nm}$. One major disadvantage of most organic NIR dyes is their very fast degradation in vivo, so longterm investigations are not feasible. To stabilize these dyes, one option is to encapsulate the dye molecules into a NP matrix.

Here, we present our recent research activities in the field of medical diagnostics concerning the encapsulation of NIR dyes into NPs for in vivo tumor diagnostics. Our work is focused on the synthesis and characterization of NP carrier systems on the basis of amorphous silica with mean particle sizes in the range of 60 to $150 \mathrm{~nm}$. These NPs are synthesized via wet-chemical synthesis and doped with different NIR dyes. The choice of silica as a basis of the NPs is motivated by their high biocompatibility, biodegradability and the possibility of surface modifications.

The characterization of the NPs is done by conventional methods such as transmission electron microscopy (TEM) and dynamic light scattering (DLS). Dye-doped NPs were characterized by fluorescence spectroscopy, measuring of absorption and emission with a plate reader and elemental analysis. The focus here was on the stability of the encapsulated NIR dyes under different storage conditions.

In summary, the synthesis of different NP systems on the basis of amorphous silica and the encapsulation of different NIR dyes was successfully demonstrated. With the confirmation of the stability of the encapsulated dyes in the NP matrix they have shown their potential in the field of medical imaging. 